\title{
Estimation of the reliability function for two-parameter exponentiated Rayleigh or Burr type X distribution
}

\author{
Anupam Pathak $^{1, *}$ and Ajit Chaturvedi ${ }^{1}$ \\ ${ }^{1}$ Department of Statistics, University of Delhi, India
}

Received: 28 October 2013; Accepted: 1 July 2014

Editor: Rahim Mahmoudvand

\begin{abstract}
Problem Statement: The two-parameter exponentiated Rayleigh distribution has been widely used especially in the modelling of life time event data. It provides a statistical model which has a wide variety of application in many areas and the main advantage is its ability in the context of life time event among other distributions. The uniformly minimum variance unbiased and maximum likelihood estimation methods are the ways to estimate the parameters of the distribution. In this study, we explore and compare the performance of the uniformly minimum variance unbiased and maximum likelihood estimators of the reliability functions $R(t)=P(X>t)$ and $P=P(X>$ $Y)$ for the two-parameter exponentiated Rayleigh distribution. Approach: A new technique of obtaining the estimators of these parametric functions is introduced in which major role is played by the estimators of powers of the parameter(s) and the functional forms of the parametric functions to be estimated are not needed. We explore the performance of these estimators numerically under varying conditions. Through the simulation study a comparison are made on the performance of these estimators with respect to the bias, mean square error (MSE), 95\% confidence length and corresponding coverage percentage. Conclusion: Based on the results of simulation study, the uniformly minimum variance unbiased estimators of $R(t)$ and ' $P$ ' for the twoparameter exponentiated Rayleigh distribution are found to be superior than maximum likelihood estimators of $R(t)$ and ' $P$ '.
\end{abstract}

Keywords Bootstrap method; Two-parameter exponentiated Rayleigh distribution; Uniformly minimum variance unbiased estimators (UMVUES);

Maximum likelihood estimators (MLES)

\section{AMS 2010 subject classifications 62Fxx}

DOI: $10.19139 /$ soic.v2i4.36

\footnotetext{
${ }^{*}$ Correspondence to: Department of Statistics, University of Delhi, Delhi 110007, India. Email: pathakanupam24@gmail.com
}

ISSN 2310-5070 (online) ISSN 2311-004X (print)

Copyright (c) 2014 International Academic Press 


\section{Introduction}

Reliability theory is mainly concerned with the determination of the probability that a system, consisting possibly of several components, will operate adequately for a given period of time in its intended application. The reliability function $R(t)$ is defined as the probability of failure-free operation until time $t$. Thus, if the random variable (rv) $X$ denotes the lifetime of an item, then $R(t)=P(X>t)$. Another measure of reliability under stress-strength set-up is the probability $P=P(X>Y)$, which represents the reliability of an item of random strength $X$ subject to random stress $Y$. Many researchers have considered the problems of estimation of $R(t)$ and ' $P$ ' for various lifetime distributions and for a brief review, one may refer to $[3,4,5,13,16,24,2,28,10,18,29,8$, 9], etc.

In [7], Burr introduced twelve different forms of cumulative distribution functions for modelling lifetime data. Among those distributions, Burr Type X and Burr Type XII are the most popular ones. Several authors considered different aspects of the Burr Type X and Burr Type XII distributions, see, for example, $[21,23,30,11,12,1,19,25]$. For an excellent review for the two distributions the readers are refereed to [15].

[26] (see also [27]) introduced two-parameter Burr Type X distribution and named as the two-parameter exponentiated Rayleigh distribution. The twoparameter exponentiated Rayleigh distribution has the following probability density function (pdf)

$$
f(x ; \alpha, \lambda)=2 \alpha \lambda^{2} x e^{-(\lambda x)^{2}}\left(1-e^{-(\lambda x)^{2}}\right)^{\alpha-1} ; \quad x, \alpha, \lambda>0
$$

and the distribution function

$$
F(x ; \alpha, \lambda)=\left\{1-e^{-(\lambda x)^{2}}\right\}^{\alpha} ; \quad x, \alpha, \lambda>0 .
$$

Here, $\alpha$ and $\lambda$ are the shape and scale parameters, respectively. In [20], the authors observed that for $\alpha \leq 1 / 2$, the pdf of two-parameter exponentiated Rayleigh distribution is a decreasing function and it is a right skewed unimodal function for $\alpha>1 / 2$. They found different forms of the density function. It is also observed that the hazard function of a two-parameter exponentiated Rayleigh distribution can be either bathtub type or an increasing function, depending on the shape parameter $\alpha$. For $\alpha \leq 1 / 2$, the hazard function of two-parameter exponentiated Rayleigh distribution is bathtub type and, for $\alpha>1 / 2$, it has an increasing hazard function. Surles and Padgett [26] showed that the two-parameter exponentiated Rayleigh distribution can be used quite effectively in modelling strength data and also modelling general lifetime data. Kundu and Raqab [17] proposed different methods of estimation for generalized Rayleigh distribution.

The rest of the study is arranged as follows. In Section 2, we derive the UMVUES of the reliability function $R(t)$ and ' $P$ ' assuming $\alpha$ to be unknown 
but $\lambda$ known. In Section 3, we obtain the MLES of the reliability function $R(t)$ and ' $P$ ', when all the parameters are unknown. In Section 4 , simulation study is carried out to investigate the performance of estimators. Finally, In Section 5, discussion is made and followed by conclusion.

\section{UMVUES of the powers of $\alpha, R(t)$ and ' $P$ ' when $\lambda$ is known}

Let $X_{1}, X_{2}, \ldots, X_{n}$ be a random sample of size $n$ from (1).

\section{Lemma 1}

Let $S=-\sum_{i=1}^{n} \ln \left\{1-e^{-\left(\lambda x_{i}\right)^{2}}\right\}$. Then, $S$ is complete and sufficient for the distribution given at (1). Moreover, the pdf of $S$ is

$$
h(s ; \alpha)=\frac{\alpha^{n}}{\Gamma(n)} s^{n-1} \exp (-\alpha s) ; \quad s>0 .
$$

\section{Proof}

From (1), the joint pdf of $X_{1}, X_{2}, \ldots, X_{n}$ is

$$
\begin{aligned}
& h\left(x_{1}, x_{2}, \ldots, x_{n} ; \alpha, \lambda\right) \\
& =\prod_{i=1}^{n} f\left(x_{i} ; \alpha, \lambda\right) \\
& =\left(2 \alpha \lambda^{2}\right)^{n}\left\{\prod_{i=1}^{n} x_{i}\right\} \exp \left\{-\lambda^{2} \sum_{i=1}^{n} x_{i}^{2}\right\} \exp \left\{(\alpha-1) \sum_{i=1}^{n} \ln \left\{1-e^{-\left(\lambda x_{i}\right)^{2}}\right\}\right\} \\
& =\left(2 \alpha \lambda^{2}\right)^{n}\left\{\prod_{i=1}^{n} x_{i}\right\} \exp \left\{-\lambda^{2} \sum_{i=1}^{n} x_{i}^{2}\right\}\left\{\prod_{i=1}^{n} \frac{1}{\left\{1-e^{-\left(\lambda x_{i}\right)^{2}}\right\}} \exp (-\alpha s) .\right.
\end{aligned}
$$

It follows from (3) and Fisher-Neymann factorization theorem [see [22], p. 341] that $S$ is sufficient for the distribution given in (1). In (1), if we make the transformation $Y=\left\{1-e^{-(\lambda X)^{2}}\right\}$, then the pdf of $Y$ is

$$
g(y ; \alpha)=\alpha y^{\alpha-1} ; \quad 0<y<1 .
$$

Letting $\ln Y=Z$, the pdf of $Z$ is

$$
g(z ; \alpha)=e^{\alpha z} ; \quad-\infty<z<0 .
$$

Further, letting, $-2 \alpha Z=V$, the pdf of $V$ is

$$
g(v ; \alpha)=\frac{1}{2} e^{-v / 2} ; \quad 0<v<\infty,
$$


which is $\chi_{(2)}^{2}$. Thus, from the additive property of gamma distribution [see [14], p. 170]

$$
2 \alpha S=-2 \alpha \sum_{i=1}^{n} \ln \left\{1-e^{-\left(\lambda X_{i}\right)^{2}} \sim \chi_{(2 n)}^{2} .\right.
$$

Hence, the distribution of $S$ follows from (4). Since the distribution of $S$ belongs to exponential family, it is also complete [see [22], p. 170].

The following lemma provides the UMVUES of the powers of $\alpha$.

\section{Theorem 1}

For $q \in(-\infty, \infty)$, the UMVUE of $\alpha^{q}$ is

$$
\hat{\alpha}^{q}= \begin{cases}\frac{\Gamma(n)}{\Gamma(n-q)} S^{-q} ; & q<n \\ 0 ; & \text { otherwise }\end{cases}
$$

Proof

From (4),

$$
\begin{aligned}
E(2 \alpha S)^{-q} & =E\left[\chi_{(2 n)}^{2}\right]^{-q} \\
& =\frac{1}{2^{n} \Gamma(n)} \int_{0}^{\infty} e^{-y / 2} y^{n-q-1} d y \\
& =\frac{\Gamma(n-q)}{2^{q} \Gamma(n)} ; \quad q<n,
\end{aligned}
$$

or,

$$
E\left[S^{-q}\left\{\frac{\Gamma(n)}{\Gamma(n-q)}\right\}\right]=\alpha^{q} .
$$

Hence, the theorem follows from Lehmann-Scheffé theorem [see [22], p. 357].

In the following lemma, we provide the UMVUE of the sampled pdf (1) at specified point ' $x$ '.

\section{Lemma 2}

The UMVUE of $f(x ; \alpha, \lambda)$ at a specified point ' $x$ ' is

$$
\begin{aligned}
& \hat{f}(x ; \alpha, \lambda) \\
& =\left\{\begin{array}{c}
2(n-1) \lambda^{2} S^{-1} x e^{-(\lambda x)^{2}}\left(1-e^{-(\lambda x)^{2}}\right)^{-1}\left(1+S^{-1} \ln \left\{1-e^{-(\lambda x)^{2}}\right\}\right)^{n-2} ; \\
-S<\ln \left\{1-e^{-(\lambda x)^{2}}\right\}, \\
0 ; \quad \text { otherwise. }
\end{array}\right.
\end{aligned}
$$




\section{Proof}

Since $S$ is complete and sufficient for the distribution $f(x ; \alpha, \lambda)$, any function $H(S)$ of $S$ satisfying $E[H(S)]=f(x ; \alpha, \lambda)$ will be the UMVUE of $f(x ; \alpha, \lambda)$. From (1) and Lemma 1, we have

$$
E[H(S)]=f(x ; \alpha, \lambda),
$$

or,

$$
\int_{0}^{\infty} H(s) \frac{\alpha^{n}}{\Gamma(n)} s^{n-1} e^{-\alpha s} d s=2 \alpha \lambda^{2} x e^{-(\lambda x)^{2}}\left\{1-e^{-(\lambda x)^{2}}\right\}^{\alpha-1}
$$

or,

$\frac{\alpha^{n}}{\Gamma(n)} \int_{0}^{\infty} H(s) s^{n-1} e^{-\alpha s} d s=2 \alpha \lambda^{2} x e^{-(\lambda x)^{2}}\left(1-e^{-(\lambda x)^{2}}\right)^{-1} \exp \left(\alpha \ln \left\{1-e^{-(\lambda x)^{2}}\right\}\right)$,

or,

$$
\begin{aligned}
& \frac{\alpha^{n}}{\Gamma(n)} \int_{0}^{\infty} H(s) s^{n-1} \exp \left[-\alpha\left(s+\ln \left\{1-e^{-(\lambda x)^{2}}\right\}\right)\right] d s \\
& \quad=2 \alpha \lambda^{2} x e^{-(\lambda x)^{2}}\left\{1-e^{-(\lambda x)^{2}}\right\}^{-1} .
\end{aligned}
$$

Let us choose

$$
H(s)=\left\{\begin{array}{c}
2(n-1) \lambda^{2} s^{-1} x e^{-(\lambda x)^{2}}\left(1-e^{-(\lambda x)^{2}}\right)^{-1}\left(1+s^{-1} \ln \left\{1-e^{-(\lambda x)^{2}}\right\}\right)^{n-2} \\
-s<\ln \left\{1-e^{-(\lambda x)^{2}}\right\}, \\
0 ; \quad \text { otherwise. }
\end{array}\right.
$$

Then,

$$
\begin{aligned}
\text { LHS of }(2.3)= & \frac{2(n-1) \alpha^{n} \lambda^{2} x e^{-(\lambda x)^{2}}\left\{1-e^{-(\lambda x)^{2}}\right\}^{-1}}{\Gamma(n)} \\
& \cdot \int_{-\ln \left\{1-e^{-(\lambda x)^{2}}\right\}}^{\infty}\left(s+\ln \left\{1-e^{-(\lambda x)^{2}}\right\}\right)^{n-2} \\
& \cdot \exp \left[-\alpha\left(s+\ln \left\{1-e^{-(\lambda x)^{2}}\right\}\right)\right] d s \\
= & \frac{2(n-1) \alpha^{n} \lambda^{2} x e^{-(\lambda x)^{2}}\left\{1-e^{-(\lambda x)^{2}}\right\}^{-1}}{\Gamma(n)} \int_{0}^{\infty} y^{n-2} \exp (-\alpha y) d y \\
= & \text { RHS. }
\end{aligned}
$$

Hence the lemma holds. 


\section{Remark 1}

We can write (1) as

$$
\begin{aligned}
f(x ; \alpha, \lambda) & =2 \alpha \lambda^{2} x e^{-(\lambda x)^{2}}\left(1-e^{-(\lambda x)^{2}}\right)^{-1} \exp \left(\alpha \ln \left\{1-e^{-(\lambda x)^{2}}\right\}\right) \\
& =2 \alpha \lambda^{2} x e^{-(\lambda x)^{2}}\left(1-e^{-(\lambda x)^{2}}\right)^{-1} \sum_{i=0}^{\infty} \frac{\left(\ln \left\{1-e^{-(\lambda x)^{2}}\right\}\right)^{i}}{i !} \alpha^{i+1} .
\end{aligned}
$$

Using (2.4), Theorem 1 and Lemma 1 of Chaturvedi and Tomer (2002), UMVUE of $f(x ; \alpha, \lambda)$ at a specified point ' $x$ ' is

$$
\begin{aligned}
& \hat{f}(x ; \alpha, \lambda) \\
& =2 \lambda^{2} x e^{-(\lambda x)^{2}}\left(1-e^{-(\lambda x)^{2}}\right)^{-1} \sum_{i=0}^{\infty} \frac{\left(\operatorname { l n } \left\{1-e^{\left.\left.-(\lambda x)^{2}\right\}\right)^{i}}\right.\right.}{i !} \hat{\alpha}^{i+1} \\
& =2 \lambda^{2} x e^{-(\lambda x)^{2}}\left(1-e^{-(\lambda x)^{2}}\right)^{-1} \sum_{i=0}^{\infty} \frac{\left(\ln \left\{1-e^{-(\lambda x)^{2}}\right\}\right)^{i}}{i !}\left\{\frac{\Gamma(n)}{\Gamma(n-i-1)}\right\} S^{-(i+1)} \\
& =2(n-1) \lambda^{2} S^{-1} x e^{-(\lambda x)^{2}}\left(1-e^{-(\lambda x)^{2}}\right)^{-1} \sum_{i=0}^{n-2}\left(\begin{array}{c}
n-2 \\
i
\end{array}\right)\left(S^{-1} \ln \left\{1-e^{-(\lambda x)^{2}}\right\}\right)^{i} \\
& =\left\{\begin{array}{c}
2(n-1) \lambda^{2} S^{-1} x e^{-(\lambda x)^{2}}\left(1-e^{-(\lambda x)^{2}}\right)^{-1}\left(1+S^{-1} \ln \left\{1-e^{-(\lambda x)^{2}}\right\}\right)^{n-2} ; \\
-S<\ln \left\{1-e^{-(\lambda x)^{2}}\right\}, \\
0 ; \quad \text { otherwise, }
\end{array}\right.
\end{aligned}
$$

which coincide with Lemma 2. Thus, the UMVUES of the powers of $\alpha$ can be used to derive the UMVUE of $f(x ; \alpha, \lambda)$ at a specified point ' $x$ '.

In the following theorem, we obtain UMVUE of $R(t)$.

\section{Theorem 2}

The UMVUE of $R(t)$ is given by

$$
\hat{R}(t)= \begin{cases}1-\left(1+S^{-1} \ln \left\{1-e^{-(\lambda t)^{2}}\right\}\right)^{n-1} ; & -S<\ln \left\{1-e^{-(\lambda t)^{2}}\right\} \\ 0 ; & \text { otherwise }\end{cases}
$$

\section{Proof}

Since $F(x, s)=f(x ; \alpha, \lambda) h(s ; \alpha)$ is a continuous function of $(X, S)$ on the rectangle $[t, \infty) \times[0, \infty)$, the conditions of Fubini's theorem [see Bilodeau et al ([6], p.207)] are satisfied for the change of order of integration. Let us consider 
the expected value of the integral $\int_{t}^{\infty} f(x ; \alpha, \lambda) d x$ with respect to $S$, i.e.,

$$
\begin{aligned}
\int_{0}^{\infty}\left\{\int_{t}^{\infty} \hat{f}(x ; \alpha, \lambda) d x\right\} h(s ; \alpha) d s & =\int_{t}^{\infty}\left[E_{S}\{\hat{f}(x ; \alpha, \lambda)\}\right] d x \\
& =\int_{t}^{\infty} f(x ; \alpha, \lambda) d x \\
& =R(t) .
\end{aligned}
$$

We conclude from (7) that the UMVUE of $R(t)$ can be obtained simply integrating $\hat{f}(x ; \alpha, \lambda)$ from $t$ to $\infty$. Thus, from Lemma 2 ,

$$
\begin{aligned}
\hat{R}(t)=2(n-1) S^{-1} \int_{t}^{\infty} \lambda^{2} x e^{-(\lambda x)^{2}}\left\{1-e^{-(\lambda x)^{2}}\right\}\left(1+S^{-1} \ln \left\{1-e^{-(\lambda x)^{2}}\right\}\right)^{n-2} d x \\
-S<\ln \left\{1-e^{-(\lambda x)^{2}}\right\} \\
=(n-1) \int_{S^{-1} \ln \left\{1-e^{-(\lambda t)^{2}}\right\}}^{0}(1+y)^{n-2} d y
\end{aligned}
$$

and the theorem follows.

Let $X$ and $Y$ be two independent rv's following the distributions $f_{1}\left(x ; \alpha_{1}, \lambda_{1}\right)$ and $f_{2}\left(y ; \alpha_{2}, \lambda_{2}\right)$, respectively, where

$$
f_{1}\left(x ; \alpha_{1}, \lambda_{1}\right)=2 \alpha_{1} \lambda_{1}^{2} x e^{-\left(\lambda_{1} x\right)^{2}}\left(1-e^{-\left(\lambda_{1} x\right)^{2}}\right)^{\alpha_{1}-1} ; \quad x>0, \alpha_{1}, \lambda_{1}>0
$$

and

$$
f_{2}\left(y ; \alpha_{2}, \lambda_{2}\right)=2 \alpha_{2} \lambda_{2}^{2} y e^{-\left(\lambda_{2} y\right)^{2}}\left(1-e^{-\left(\lambda_{2} y\right)^{2}}\right)^{\alpha_{2}-1} ; \quad y>0, \alpha_{2}, \lambda_{2}>0 .
$$

Here, we assume that $\alpha_{1}$ and $\alpha_{2}$ are unknown but $\lambda_{1}$ and $\lambda_{2}$ are known. Let $X_{1}, X_{2}, \ldots, X_{n}$ be a random sample of size $n$ from $f_{1}\left(x ; \alpha_{1}, \lambda_{1}\right)$ and $Y_{1}, Y_{2}, \ldots, Y_{m}$ be a random sample of size $m$ from $f_{2}\left(y ; \alpha_{2}, \lambda_{2}\right)$. Let us denote by $S=-\sum_{i=1}^{n} \ln \left\{1-e^{-\left(\lambda_{1} x_{i}\right)^{2}}\right\}$ and $T=-\sum_{j=1}^{m} \ln \left\{1-e^{-\left(\lambda_{2} y_{j}\right)^{2}}\right\}$.

In what follows, we obtain the UMVUE of ' $P$ '. 


\section{Theorem 3}

The UMVUE of ' $P$ ' is given by

$$
\hat{P}=\left\{\begin{array}{c}
1-(m-1) \int_{0}^{1}\left(1+S^{-1} \ln \left\{1-\left(1-e^{-T v}\right)^{\lambda_{1}^{2} / \lambda_{2}^{2}}\right\}^{n-1}(1-v)^{m-2} d v\right. \\
{\left[-\lambda_{2}^{-2} \ln \left(1-e^{-T}\right)\right]^{1 / 2}>\left[-\lambda_{1}^{-2} \ln \left(1-e^{-S}\right)\right]^{1 / 2}} \\
1-(m-1) \int_{0}^{-S^{-1} \ln \left\{1-\left(1-e^{-S}\right)^{\lambda_{2}^{2} / \lambda_{1}^{2}}\right\}}\left(1+S^{-1} \ln \left\{1-\left(1-e^{-T v}\right)^{\lambda_{1}^{2} / \lambda_{2}^{2}}\right\}\right)^{n-1} \\
{\left[-\lambda_{2}^{-2} \ln \left(1-e^{-T}\right)\right]^{1 / 2}<\left[-\lambda_{1}^{-2} \ln \left(1-e^{-S}\right)\right]^{1 / 2} \cdot}
\end{array}\right.
$$

Proof

It follows from Lemma 2 that the UMVUES of $f_{1}\left(x ; \alpha_{1}, \lambda_{1}\right)$ and $f_{2}\left(y ; \alpha_{2}, \lambda_{2}\right)$ at specified points ' $x$ ' and ' $y$ ', respectively, are

$$
\hat{f}_{1}\left(x ; \alpha_{1}, \lambda_{1}\right)=\left\{\begin{array}{c}
2(n-1) \lambda_{1}^{2} S^{-1} x e^{-\left(\lambda_{1} x\right)^{2}} \\
\cdot\left(1-e^{-\left(\lambda_{1} x\right)^{2}}\right)^{-1}\left(1+S^{-1} \ln \left\{1-e^{-\left(\lambda_{1} x\right)^{2}}\right\}\right)^{n-2} \\
\quad-S<\ln \left\{1-e^{-\left(\lambda_{1} x\right)^{2}}\right\} \\
0 ; \quad \text { otherwise. }
\end{array}\right.
$$

and

$$
\hat{f}_{2}\left(y ; \alpha_{2}, \lambda_{2}\right)=\left\{\begin{array}{cl}
2(m-1) \lambda_{2}^{2} T^{-1} x e^{-\left(\lambda_{2} y\right)^{2}} \\
\cdot\left(1-e^{-\left(\lambda_{2} x\right)^{2}}\right)^{-1}\left(1+T^{-1} \ln \left\{1-e^{-\left(\lambda_{2} y\right)^{2}}\right\}\right)^{m-2} \\
\quad-T<\ln \left\{1-e^{-\left(\lambda_{2} y\right)^{2}}\right\} \\
0 ; \quad \text { otherwise. }
\end{array}\right.
$$

From the arguments similar to those adopted in the proof of Theorem 2, it can be shown that the UMVUE of ' $P$ ' is given by

$$
\begin{aligned}
\hat{P} & =\int_{y=0}^{\infty} \int_{x=y}^{\infty} \hat{f}_{1}\left(x ; \alpha_{1}, \lambda_{1}\right) \hat{f}_{2}\left(y ; \alpha_{2}, \lambda_{2}\right) d x d y \\
& =\int_{y=0}^{\infty} \hat{R}_{1}\left(y ; \alpha_{1}, \lambda_{1}\right) \hat{f}_{2}\left(y ; \alpha_{2}, \lambda_{2}\right) d y .
\end{aligned}
$$

Stat., Optim. Inf. Comput. Vol. 2, December 2014. 
Applying Lemma 2 and Theorem 2, we get

$$
\begin{aligned}
& \hat{P}=1-(m-1) \lambda_{2} T^{-1} \\
& \int_{y=\max \left\{\left[-\lambda_{2}^{-2} \ln \left(1-e^{-T}\right)\right]^{1 / 2},\left[-\lambda_{1}^{-2} \ln \left(1-e^{-S}\right)\right]^{1 / 2}\right\}}^{\infty}\left\{1+S^{-1} \ln \left\{1-e^{-\left(\lambda_{1} y\right)^{2}}\right\}\right\}^{n-1} \\
& \cdot \lambda_{2}^{2} x e^{-\left(\lambda_{2} y\right)^{2}}\left(1-e^{-\left(\lambda_{2} x\right)^{2}}\right)\left\{1+T^{-1} \ln \left\{1-e^{-\left(\lambda_{2} y\right)^{2}}\right\}\right\}^{m-2} d y .
\end{aligned}
$$

Let us first consider the case when $\left[-\lambda_{2}^{-2} \ln \left(1-e^{-T}\right)\right]^{1 / 2}>\left[-\lambda_{1}^{-2} \ln (1-\right.$ $\left.\left.e^{-S}\right)\right]^{1 / 2}$. In this case, from (10),

$$
\hat{P}=1-(m-1) \int_{0}^{1}\left(1+S^{-1} \ln \left\{1-\left(1-e^{-T v}\right)^{\lambda_{1}^{2} / \lambda_{2}^{2}}\right\}\right)^{n-1}(1-v)^{m-2} d v
$$

Now, we consider the case when $\left[-\lambda_{2}^{-2} \ln \left(1-e^{-T}\right)\right]^{1 / 2}<\left[-\lambda_{1}^{-2} \ln \left(1-e^{-S}\right)\right]^{1 / 2}$. In this case, from (10),

$$
\begin{aligned}
\hat{P}= & 1-(m-1) \\
& \times\left(1+S^{-1} \ln \left\{1-\left(1-e^{-T v}\right)^{\lambda_{1}^{2} / \lambda_{2}^{2}}\right\}\right)^{n-1}(1-v)^{m-2} d v .
\end{aligned}
$$

The theorem now follows on combining (11) and (12).

\section{Corollary 1}

In the case when $\lambda_{1}=\lambda_{2}=\lambda$, say,

$$
\hat{P}= \begin{cases}1-\sum_{i=0}^{n-1}(-1)^{i} \frac{(n-1) !(m-1) !}{(n-i-1) !(m+i-1) !}\left(\frac{T}{S}\right)^{i} ; & S<T \\ 1-\sum_{j=0}^{m-2}(-1)^{j} \frac{(n-1) !(m-1) !}{(n+j) !(m-j-2) !}\left(\frac{S}{T}\right)^{j+1} ; & S>T\end{cases}
$$

Stat., Optim. Inf. Comput. Vol. 2, December 2014. 


\section{Proof}

From Theorem 3, for $S<T$,

$$
\begin{aligned}
\hat{P} & =1-(m-1) \int_{1}^{0}\left(1-\frac{T}{S} v\right)^{n-1}(1-v)^{m-2} d v \\
& =1-(m-1) \int_{0}^{1} \sum_{i=0}^{n-1}(-1)^{i}\left(\begin{array}{c}
n-1 \\
i
\end{array}\right)\left(\frac{T}{S} v\right)^{i}(1-v)^{m-2} d v \\
& =1-(m-1) \sum_{i=0}^{n-1}(-1)^{i}\left(\begin{array}{c}
n-1 \\
i
\end{array}\right)\left(\frac{T}{S}\right)^{i} \int_{0}^{1} v^{i+1-1}(1-v)^{m-2} d v \\
& =1-(m-1) \sum_{i=0}^{n-1}(-1)^{i}\left(\frac{T}{S}\right)^{i} B(m-1, i+1) \\
& =1-\sum_{i=0}^{n-1}(-1)^{i} \frac{(n-1) !(m-1) !}{(n-i-1) !(m+i-1) !}\left(\frac{T}{S}\right)^{i}
\end{aligned}
$$

and the first assertion follows. From Theorem 3, for $S>T$,

$$
\begin{aligned}
\hat{P} & =1-(m-1) \int_{S / T}^{0}\left(1-\frac{T}{S} v\right)^{n-1}(1-v)^{m-2} d v \\
& =1-(m-1) \int_{1}^{0}(1-w)^{n-1}\left(1-\frac{S}{T} w\right)^{m-2}\left(\frac{S}{T}\right) v^{j+1-1}(1-v)^{m-2} d w \\
& =1-(m-1) \int_{1}^{0}(1-w)^{n-1} \sum_{j=0}^{m-2}(-1)^{j}\left(\begin{array}{c}
m-2 \\
j
\end{array}\right)\left(\frac{S}{T}\right)^{j}(w)^{j}\left(\frac{S}{T}\right) d w \\
& =1-(m-1) \sum_{j=0}^{m-2}(-1)^{j}\left(\begin{array}{c}
m-2 \\
j
\end{array}\right)\left(\frac{S}{T}\right)^{j+1} B(n, j+1) \\
& =1-\sum_{j=0}^{m-2}(-1)^{j} \frac{(n-1) !(m-1) !}{(n+j) !(m-j-2) !}\left(\frac{S}{T}\right)^{j+1}
\end{aligned}
$$

and the second assertion follows.

\section{Remark 2}

It follows from Theorem 1 that $V(\hat{\alpha})=\frac{\alpha^{2}}{n-2} \rightarrow 0$ as $n \rightarrow \infty$. Thus, $\hat{\alpha}$ is a 
consistent estimator of $\alpha$. Since $\hat{f}(x ; \alpha, \lambda), \hat{R}(t)$ and $\hat{P}$ are continuous functions of consistent estimators, therefore, they are also consistent estimators.

\section{MLES of $R(t)$ and ' $P$ ' when all the parameters are unknown}

Following the lines of derivations in [17], it can be shown that the MLES of $\alpha$ and $\lambda$ are solutions of

$$
\tilde{\alpha}(\lambda)=-\frac{n}{\sum_{i=1}^{n} \ln \left(1-e^{-\left(\lambda x_{i}\right)^{2}}\right)}
$$

and

$$
\tilde{\lambda}=\left[\frac{\sum_{i=1}^{n} \frac{x_{i}^{2} e^{-\left(\lambda x_{i}\right)^{2}}}{\left(1-e^{-\left(\lambda x_{i}\right)^{2}}\right)}}{\sum_{i=1}^{n} \ln \left(1-e^{-\left(\lambda x_{i}\right)^{2}}\right)}+\frac{1}{n} \sum_{i=1}^{n} x_{i}^{2}+\frac{1}{n} \sum_{i=1}^{n} \frac{x_{i}^{2} e^{-\left(\lambda x_{i}\right)^{2}}}{\left(1-e^{-\left(\lambda x_{i}\right)^{2}}\right)}\right]^{-1},
$$

respectively.

From one-to-one property, MLES of $f(x ; \alpha, \lambda), R(t)$ and ' $P$ ' are given, respectively, by $\tilde{f}(x ; \alpha, \lambda), \tilde{R}(t)$ and $\tilde{P}$, where

$$
\begin{aligned}
\tilde{f}(x ; \alpha, \lambda) & =2 \tilde{\alpha} \tilde{\lambda}^{2} x e^{-\left(\tilde{\lambda} x^{2}\right)}\left(1-e^{-(\tilde{\lambda} x)^{2}}\right)^{\tilde{\alpha}-1}, \\
\tilde{R}(t) & =\int_{t}^{\infty} \tilde{f}(x ; \alpha, \lambda) d x \\
& =2 \tilde{\alpha} \tilde{\lambda}^{2} \int_{t}^{\infty} x e^{-\left(\tilde{\lambda} x^{2}\right)}\left(1-e^{-(\tilde{\lambda} x)^{2}}\right)^{\tilde{\alpha}-1} d x=\tilde{\alpha} \int_{\left(1-e^{\left.-(\tilde{\lambda} t)^{2}\right)}\right.}^{1} u^{\tilde{\alpha}-1} d u \\
& =1-\left\{1-e^{-(\tilde{\lambda} t)^{2}}\right\}^{\tilde{\alpha}}
\end{aligned}
$$


and

$$
\begin{aligned}
\tilde{P} & =\int_{y=0}^{\infty} \int_{x=y}^{\infty} \tilde{f}_{1}\left(x ; \alpha_{1}, \lambda_{1}\right) \tilde{f}_{2}\left(y ; \alpha_{2}, \lambda_{2}\right) d x d y \\
& =\int_{0}^{\infty}\left(1-\tilde{R}_{2}(x)\right) \tilde{f}_{1}\left(x ; \alpha_{1}, \lambda_{1}\right) d x \\
& =2 \tilde{\alpha}_{1} \tilde{\lambda}_{1}^{2} \int_{0}^{\infty}\left[1-e^{-\left(\tilde{\lambda}_{2} x\right)^{2}}\right]^{\tilde{\alpha}_{2}} x^{2} e^{-\left(\tilde{\lambda}_{1} x^{2}\right)}\left(1-e^{-\left(\tilde{\lambda}_{1} x\right)^{2}}\right)^{\tilde{\alpha}_{1}-1} d x \\
& =\tilde{\alpha}_{1} \int_{0}^{1}\left[1-(1-u)^{\tilde{\lambda}_{2}^{2} / \tilde{\lambda}_{1}^{2}}\right]^{\tilde{\alpha}_{2}} u^{\tilde{\alpha}_{1}-1} d u
\end{aligned}
$$

It can also be easily verified that when $\lambda_{1}=\lambda_{2}=\lambda$, say,

$$
\tilde{P}=\frac{\tilde{\alpha}_{1}}{\tilde{\alpha}_{1}+\tilde{\alpha}_{2}} \text {. }
$$

Remarks 1(i) In the literature, the researchers have dealt with the estimation of $R(t)$ and ' $P$ ', separately. If we look at the proof of Theorems 2 and 3, we observe that the UMVUE of the sampled pdf is used to obtain the UMVUES of $R(t)$ and ' $P$ ', respectively, which is also true for MLES. Thus we have established interrelationship between the two estimation problems. Moreover, in the present approach, one does not require the expressions of $R(t)$ and ' $P$ '.

(ii) Since the UMVUES and MLES of powers of $\alpha$ are obtained under same conditions, we compare their performances. For $q=-1$ the UMVUE and MLE of $\alpha$ are, respectively $\hat{\alpha}=(n-1)(-T)^{-1}$ and $\tilde{\alpha}=(n)(-T)^{-1}$. For these estimators,

$$
V(\hat{\alpha})=\frac{\alpha^{2}}{n-2} \quad \text { and } \quad V(\tilde{\alpha})=\frac{n^{2} \alpha^{2}}{(n-1)^{2}(n-2)} .
$$

Thus,

$$
V(\tilde{\alpha})-V(\hat{\alpha})=\frac{(2 n-1)}{(n-1)(n-2)} \alpha^{2}>0 .
$$

Thus, the UMVUE of $\alpha$ is more efficient than its MLE. Similarly, we can compare the performances of these estimators for other powers of $\alpha$. 


\section{Numerical Findings}

In order to compare the efficiency of the estimators $\hat{\alpha}$ and $\tilde{\alpha}$, when $\lambda$ is known, we have calculated variances of $\hat{\alpha}$ and $\tilde{\alpha}$, for samples of sizes $n=5,10,20,30$ and 50 corresponding to $\alpha=0.80(0.60) 4.20$ and these results are reported in Table 1 . From Table 1, it is clear that $\hat{\alpha}$ is more efficient than $\tilde{\alpha}$.

Table I

\begin{tabular}{|c|c|c|c|c|c|c|c|c|c|c|}
\hline $\mathbf{n}$ & \multicolumn{2}{|c|}{$\mathbf{5}$} & \multicolumn{2}{c|}{$\mathbf{1 0}$} & \multicolumn{2}{c|}{$\mathbf{2 0}$} & \multicolumn{2}{|c|}{$\mathbf{3 0}$} & \multicolumn{2}{c|}{$\mathbf{5 0}$} \\
\hline$\alpha$ & $v(\hat{\alpha})$ & $v(\tilde{\alpha})$ & $v(\hat{\alpha})$ & $v(\tilde{\alpha})$ & $v(\hat{\alpha})$ & $v(\tilde{\alpha})$ & $v(\hat{\alpha})$ & $v(\tilde{\alpha})$ & $v(\hat{\alpha})$ & $v(\tilde{\alpha})$ \\
\hline 0.80 & 0.2133 & 0.3333 & 0.0800 & 0.0988 & 0.0356 & 0.0394 & 0.0229 & 0.0245 & 0.0133 & 0.0139 \\
\hline 1.40 & 0.6533 & 1.0208 & 0.2450 & 0.3025 & 0.1089 & 0.1207 & 0.0700 & 0.0749 & 0.0408 & 0.0425 \\
\hline 2.00 & 1.3333 & 2.0833 & 0.5000 & 0.6173 & 0.2222 & 0.2462 & 0.1429 & 0.1529 & 0.0833 & 0.0868 \\
\hline 2.60 & 2.2533 & 3.5208 & 0.8450 & 1.0432 & 0.3756 & 0.4161 & 0.2414 & 0.2584 & 0.1408 & 0.1466 \\
\hline 3.20 & 3.4133 & 5.3333 & 1.2800 & 1.5802 & 0.5689 & 0.6303 & 0.3657 & 0.3914 & 0.2133 & 0.2221 \\
\hline 3.80 & 4.8133 & 7.5208 & 1.8050 & 2.2284 & 0.8022 & 0.8889 & 0.5157 & 0.5519 & 0.3008 & 0.3132 \\
\hline 4.20 & 5.8800 & 9.1875 & 2.2050 & 2.7222 & 0.9800 & 1.0859 & 0.6300 & 0.6742 & 0.3675 & 0.3827 \\
\hline
\end{tabular}

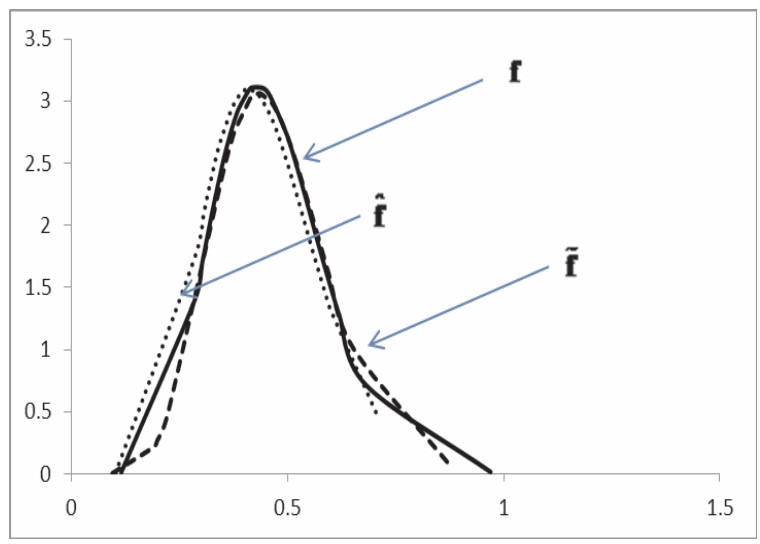

Figure 1. Curves of $f(x ; \alpha, \lambda), \hat{f}(x ; \alpha, \lambda)$ and $\tilde{f}(x ; \alpha, \lambda)$.

In order to verify the consistency of the estimators obtained, we have drawn sample of sizes $n=30$ from (1), with $\alpha=4$ and $\lambda=3$. In Fig. 1, we have plotted $f(x ; \alpha, \lambda), \hat{f}(x ; \alpha, \lambda)$ and $\tilde{f}(x ; \alpha, \lambda)$, respectively, corresponding to this sample. We conclude from Fig. 1 that curves of $\hat{f}(x ; \alpha, \lambda)$ and $\tilde{f}(x ; \alpha, \lambda)$ overlap to the curve of $f(x ; \alpha, \lambda)$ for $n=30$. This justifies the consistency property of the estimators.

In order to demonstrate the application of the theory developed in Section 3, we generated a sample of size $n=30$ from (1) for $\alpha=4$ and $\lambda=3$. Solving (13) and 
Table II. Simulation results for $R(t)$.

\begin{tabular}{|c|c|c|c|c|c|c|c|c|c|}
\hline \multirow[t]{2}{*}{$\mathbf{t}$} & \multirow{2}{*}{$\begin{array}{c}\mathbf{n} \\
R(t)\end{array}$} & \multicolumn{2}{|c|}{5} & \multicolumn{2}{|c|}{10} & \multicolumn{2}{|c|}{20} & \multicolumn{2}{|c|}{50} \\
\hline & & $\hat{R}(t)$ & $\tilde{R}(t)$ & $\hat{R}(t)$ & $\tilde{R}(t)$ & $\hat{R}(t)$ & $\tilde{R}(t)$ & $\hat{R}(t)$ & $\tilde{R}(t)$ \\
\hline \multirow{5}{*}{0.21} & 0.9860 & 0.9710 & 0.9539 & 0.9862 & 0.9780 & 0.9840 & 0.9804 & 0.9872 & 0.9858 \\
\hline & & -0.0151 & -0.0321 & $2 e-04$ & -0.0080 & -0.0020 & -0.0056 & 0.0011 & $-2 \mathrm{e}-04$ \\
\hline & & 0.004515932 & 0.001552421 & $8.92585 \mathrm{e}-05$ & 0.0001760942 & 0.0001253313 & 0.0001671481 & $3.53743 \mathrm{e}-05$ & $3.64641 \mathrm{e}-05$ \\
\hline & & 0.0792 & 0.0830 & 0.0368 & 0.0417 & 0.0420 & 0.0450 & 0.0228 & 0.0236 \\
\hline & & 44.9490 & 92.3063 & 89.4167 & 92.2201 & 90.6114 & 92.2691 & 92.9964 & 93.3348 \\
\hline \multirow{5}{*}{0.35} & 0.8469 & 0.8195 & 0.8278 & 0.8659 & 0.8650 & 0.8511 & 0.8515 & 0.8485 & 0.8483 \\
\hline & & -0.0274 & -0.0191 & 0.0190 & 0.0181 & 0.0041 & 0.0046 & 0.0016 & 0.0013 \\
\hline & & 0.01147278 & 0.005809513 & 0.003760792 & 0.003122649 & 0.002368198 & 0.002151073 & 0.001233167 & 0.001281566 \\
\hline & & 0.3232 & 0.2760 & 0.2257 & 0.2057 & 0.1870 & 0.1782 & 0.1379 & 0.1406 \\
\hline & & 88.0252 & 93.6421 & 94.5918 & 94.7441 & 94.4698 & 94.4954 & 95.0602 & 95.0570 \\
\hline \multirow{5}{*}{0.45} & 0.63 & 0.5396 & 0.5833 & 0.5620 & 0.5829 & 0.6232 & 0.6324 & 0.6351 & 0.6387 \\
\hline & & -0.0904 & -0.0467 & -0.0680 & -0.0471 & -0.0067 & 0.0024 & 0.0051 & 0.0087 \\
\hline & & 0.0172604 & 0.009738878 & 0.01090285 & 0.007991645 & 0.004080048 & 0.003829112 & 0.002163839 & 0.002168776 \\
\hline & & 0.3895 & 0.3516 & 0.3002 & 0.2883 & 0.2489 & 0.2423 & 0.1786 & 0.1767 \\
\hline & & 93.4056 & 93.9734 & 93.8667 & 94.0039 & 94.6170 & 94.6657 & 94.6296 & 94.6341 \\
\hline \multirow{5}{*}{0.50} & 0.5062 & 0.4364 & 0.4850 & 0.5071 & 0.5297 & 0.5007 & 0.5121 & 0.4987 & 0.5032 \\
\hline & & -0.0698 & -0.0212 & $8 \mathrm{e}-04$ & 0.0234 & -0.0055 & 0.0058 & -0.0075 & -0.0030 \\
\hline & & 0.01229542 & 0.007300615 & 0.005458611 & 0.005697628 & 0.002781147 & 0.002724515 & 0.001556892 & 0.00149941 \\
\hline & & 0.3092 & 0.2996 & 0.2847 & 0.2764 & 0.2138 & 0.2112 & 0.1567 & 0.1561 \\
\hline & & 91.0011 & 91.7419 & 92.8672 & 93.0910 & 93.4982 & 93.5788 & 95.3514 & 95.3630 \\
\hline \multirow{5}{*}{0.55} & 0.388 & 0.3073 & 0.3536 & 0.4323 & 0.4559 & 0.3366 & 0.3476 & 0.3907 & 0.3952 \\
\hline & & -0.0807 & -0.0344 & 0.0444 & 0.0679 & -0.0514 & -0.0404 & 0.0027 & 0.0073 \\
\hline & & 0.01107912 & 0.006118383 & 0.00668347 & 0.009234135 & 0.004913982 & 0.003954968 & 0.001140717 & 0.001191856 \\
\hline & & 0.2710 & 0.2814 & 0.2631 & 0.2611 & 0.1873 & 0.1895 & 0.1319 & 0.1322 \\
\hline & & 91.6221 & 92.2515 & 92.9936 & 93.2361 & 94.6500 & 94.6966 & 94.7881 & 94.7959 \\
\hline \multirow{5}{*}{0.60} & 0.284 & 0.1920 & 0.2279 & 0.2510 & 0.2605 & 0.2515 & 0.2610 & 0.2753 & 0.2713 \\
\hline & & -0.0920 & -0.0561 & -0.0331 & -0.0236 & -0.0326 & -0.0230 & -0.0088 & -0.0127 \\
\hline & & 0.01069158 & 0.005908907 & 0.002508747 & 0.00203283 & 0.002159625 & 0.00167466 & 0.0008977123 & 0.0009683548 \\
\hline & & 0.1980 & 0.2206 & 0.1516 & 0.1549 & 0.1329 & 0.1358 & 0.1173 & 0.1163 \\
\hline & & 92.9102 & 93.3796 & 94.5525 & 94.5972 & 94.6282 & 94.6683 & 95.7776 & 95.7717 \\
\hline \multirow{5}{*}{0.65} & 0.1991 & 0.2473 & 0.2890 & 0.1691 & 0.1842 & 0.2065 & 0.2149 & 0.1943 & 0.1974 \\
\hline & & 0.0482 & 0.0899 & -0.0299 & -0.0149 & 0.0074 & 0.0158 & -0.0048 & -0.0016 \\
\hline & & 0.006273621 & 0.01268783 & 0.002110118 & 0.001602851 & 0.001165438 & 0.001423038 & 0.0007551275 & 0.0007516422 \\
\hline & & 0.2530 & 0.2729 & 0.1395 & 0.1486 & 0.1305 & 0.1341 & 0.1086 & 0.1098 \\
\hline & & 92.7658 & 93.2679 & 94.0162 & 94.1039 & 94.1657 & 94.1959 & 95.1725 & 95.1831 \\
\hline
\end{tabular}

Here, the first row indicates the estimate, the second row indicates the bias, the third row indicates variance, the fourth row indicates $95 \%$ bootstrap confidence length and the fifth row indicates the coverage percentage.

Stat., Optim. Inf. Comput. Vol. 2, December 2014. 
Table III. Simulation results for ' $P$ '.

\begin{tabular}{|c|c|c|c|c|c|c|c|c|}
\hline$\left(\alpha_{1}, \alpha_{2}\right)$ & \multicolumn{2}{|c|}{$(2,1)$} & \multicolumn{2}{|c|}{$(2,3)$} & \multicolumn{2}{|c|}{$(2,4)$} & \multicolumn{2}{|c|}{$(2,5)$} \\
\hline$P$ & \multicolumn{2}{|c|}{0.6666667} & \multicolumn{2}{|c|}{0.4} & \multicolumn{2}{|c|}{$(0.3333333)$} & \multicolumn{2}{|c|}{$(0.2857143)$} \\
\hline$(n, m)$ & $\hat{P}$ & $\tilde{P}$ & $\hat{P}$ & $\tilde{P}$ & $\hat{P}$ & $\tilde{P}$ & $\hat{P}$ & $\tilde{P}$ \\
\hline \multirow{5}{*}{$(5,5)$} & 0.6528 & 0.6370 & 0.4427 & 0.4493 & 0.3154 & 0.3329 & 0.2657 & 0.2858 \\
\hline & -0.0139 & -0.0297 & 0.0427 & 0.0493 & -0.0179 & $-5 e-04$ & -0.0200 & $1 \mathrm{e}-04$ \\
\hline & 0.007232074 & 0.006763495 & 0.009493904 & 0.008494557 & 0.01030564 & 0.008504289 & 0.008154158 & 0.007051987 \\
\hline & 0.3046 & 0.2804 & 0.3215 & 0.2864 & 0.3986 & 0.3683 & 0.3375 & 0.3214 \\
\hline & 93.0223 & 93.2512 & 92.6982 & 92.8389 & 92.9623 & 93.6543 & 93.0178 & 93.5448 \\
\hline \multirow{5}{*}{$(10,5)$} & 0.6914 & 0.6672 & 0.4054 & 0.4013 & 0.3254 & 0.3271 & 0.2796 & 0.2835 \\
\hline & 0.0247 & $5 e-04$ & 0.0054 & 0.0013 & -0.0080 & -0.0062 & -0.0061 & -0.0022 \\
\hline & 0.006541987 & 0.005484255 & 0.006334268 & 0.005335373 & 0.005240716 & 0.004533083 & 0.008068555 & 0.00717309 \\
\hline & 0.2972 & 0.2861 & 0.2921 & 0.2688 & 0.2768 & 0.2582 & 0.3345 & 0.3174 \\
\hline & 93.8905 & 94.1889 & 92.9533 & 93.0341 & 93.7132 & 93.9067 & 93.2983 & 93.5995 \\
\hline \multirow{5}{*}{$(10,10)$} & 0.6590 & 0.6515 & 0.3835 & 0.3892 & 0.3264 & 0.3344 & 0.2732 & 0.2825 \\
\hline & -0.0077 & -0.0152 & -0.0165 & -0.0108 & -0.0069 & 0.0011 & -0.0125 & -0.0032 \\
\hline & 0.004755181 & 0.004579777 & 0.005455311 & 0.004852003 & 0.004134199 & 0.00381056 & 0.005270595 & 0.004904695 \\
\hline & 0.2652 & 0.2551 & 0.2736 & 0.2620 & 0.2470 & 0.2386 & 0.2724 & 0.2672 \\
\hline & 93.9416 & 94.0369 & 94.2555 & 94.3005 & 94.4152 & 94.4906 & 94.2250 & 94.3466 \\
\hline \multirow{5}{*}{$(20,20)$} & 0.6676 & 0.6638 & 0.3909 & 0.3935 & 0.3315 & 0.3352 & 0.2863 & 0.2906 \\
\hline & $9 \mathrm{e}-04$ & -0.0029 & -0.0091 & -0.0065 & -0.0018 & 0.0019 & $6 e-04$ & 0.0049 \\
\hline & 0.003312423 & 0.003204202 & 0.003548195 & 0.003359474 & 0.004023466 & 0.003890517 & 0.003917004 & 0.003850783 \\
\hline & 0.2276 & 0.2236 & 0.2243 & 0.2196 & 0.2447 & 0.2407 & 0.2392 & 0.2367 \\
\hline & 94.4945 & 94.5432 & 94.3049 & 94.3128 & 94.5514 & 94.5784 & 94.3957 & 94.4237 \\
\hline \multirow{5}{*}{$(25,25)$} & 0.6755 & 0.6724 & 0.4079 & 0.4097 & 0.3247 & 0.3277 & 0.2777 & 0.2813 \\
\hline & 0.0088 & 0.0057 & 0.0079 & 0.0097 & -0.0086 & -0.0056 & -0.0080 & -0.0045 \\
\hline & 0.002752934 & 0.002639899 & 0.003348106 & 0.003261242 & 0.00354906 & 0.003422819 & 0.002955221 & 0.002866375 \\
\hline & 0.1996 & 0.1971 & 0.2222 & 0.2182 & 0.2283 & 0.2254 & 0.2090 & 0.2072 \\
\hline & 94.6152 & 94.6283 & 94.4863 & 94.5019 & 94.5404 & 94.546 & 94.5103 & 94.5236 \\
\hline \multirow{5}{*}{$(50,50)$} & 0.6651 & 0.6636 & 0.4039 & 0.4049 & 0.3358 & 0.3372 & 0.2880 & 0.2898 \\
\hline & -0.0016 & -0.0030 & 0.0039 & 0.0049 & 0.0024 & 0.0039 & 0.0023 & 0.0040 \\
\hline & 0.001970162 & 0.001949933 & 0.002048208 & 0.002021451 & 0.002406106 & 0.002383653 & 0.002031045 & 0.002023235 \\
\hline & 0.1748 & 0.1736 & 0.1797 & 0.1781 & 0.1925 & 0.1913 & 0.1770 & 0.1761 \\
\hline & 95.0227 & 95.0298 & 95.3579 & 95.3584 & 95.0372 & 95.0412 & 95.0097 & 95.0157 \\
\hline
\end{tabular}

Here, the first row indicates the estimate, the second row indicates the bias, the third row indicates variance, the fourth row indicates $95 \%$ bootstrap confidence length and the fifth row indicates the coverage percentage.

(14) simultaneously, we get $\tilde{\alpha}=3.906139$ and $\tilde{\lambda}=2.950872$. It can be seen that $-2 \ln L=-36.5843, R(0.20)=0.9916$ and $\tilde{R}(0.20)=0.9916$.

Stat., Optim. Inf. Comput. Vol. 2, December 2014. 
In order to obtain the MLE of ' $P$ ', we have generated one more sample of size $m=30$ from (1) for $\alpha=2.5$ and $\lambda=3$. Solving as above, we get $\tilde{\lambda}=2.486173$, $\tilde{\lambda}=2.963452$ and $-2 \ln L=-32.0487$. Using this population as $Y$ and above population as $X$, we get $P=0.6153846$ and $\tilde{P}=0.6146800$.

For the case when $\alpha$ is unknown but $\lambda$ is known, we have conducted simulation experiments using bootstrap re-sampling technique for sample sizes $n=5,10,20$ and 50. The samples are generated from (1), with $\alpha=3$ and $\lambda=2.5$. For different values of $t$, we have computed $\hat{R}(t), \tilde{R}(t)$, their corresponding bias, variance, $95 \%$ confidence length and corresponding coverage percentage. All the computations are based on 500 bootstrap replications and results are reported in Table 2.

In order to estimate ' $P$ ', for the case when $\alpha_{1}$ and $\alpha_{2}$ are unknown but other parameters are known, we have conducted simulation experiments using bootstrap re-sampling technique for sample sizes $(n, m)=$ $(5,5),(10,5),(10,10),(20,20),(25,25)$ and $(50,50)$. The samples are generated from (1), with, $\alpha_{1}=2.0, \lambda_{1}=2.5$ and $\lambda_{2}=2.5$ and $\alpha_{2}=1,3(1) 5$. The computations are based on 500 bootstrap replications. We have computed $\hat{P}, \tilde{P}$, bias, variance, $95 \%$ confidence length and corresponding coverage percentage. The results are presented in Table 3.

\section{Discussion and Conclusion}

In Table 1, we compared UMVUE and MLE of $\alpha$, keeping $\lambda$ to be constant for two-parameter exponentiated Rayleigh distribution. The table shows that UMVUE of $\alpha$ is more efficient than MLE of $\alpha$. From table we observe that as we increase the sample size variance of estimators of $\alpha$ decrease (for both of estimators UMVUE as well as for MLE). Table 1 also shows that as we increase values of the parameter $\alpha$, variance increases corresponding to both of the estimators.

With the help of Fig. 1, we justified the consistency property of the estimators.

Through Table 2, we compared the efficiency of $\hat{R}(t)$ and $\tilde{R}(t)$. Table 2 shows that UMVUE of $R(t)$ is more efficient than MLE of $R(t)$. It is also clear that as we increase sample size Biasness, MSE and Confidence Length decreases but on the other hand corresponding Coverage Percentage increases. These statements are also true for the estimators $\hat{P}$ and $\tilde{P}$.

\section{Acknowledgement}

Authors are thankful to the referee for his valuable comments and first author acknowledge his deep sense of gratitude to Dr. Ajit Chaturvedi, who has been a constant source of inspiration and affectionate guidance during the research work. 


\section{REFERENCES}

1. K. E. Ahmad, M. E. Fakhry and Z. F. Jaheen, Empirical Bayes estimation of $P(Y<X)$ and characterizations of the Burr type X model, Jour. Statist. Plann. Infer., 64, 297 - 308 (1997).

2. A. M. Awad and M. K. Gharraf, Estimation of $P(Y<X)$ in the Burr case: A Comparative Study, Commun. Statist. - Simul., 15 (2), 389 - 403 (1986).

3. D. J. Bartholomew, A problem in life testing, Jour. Amer. Statist. Assoc., 52, 350 - 355 (1957).

4. D.J. Bartholomew, The sampling distribution of an estimate arising in life testing, Technometrics, 5, $361-374$ (1963).

5. A. P. Basu, Estimates of reliability for some distributions useful in life testing, Technometrics, 6, $215-219$ (1964).

6. G. G. Bilodeau, P. R. Thie and G. F. Keough, An Introduction to Analysis, Jones and Bartlett Learning International, U.K. (2010).

7. I. W. Burr, Cumulative frequency functions, Ann. Math. Statist., 13, 215 - 232 (1942).

8. A. Chaturvedi and U. Rani, Classical and Bayesian reliability estimation of the generalized Maxwell failure distribution, Jour. Statist. Res., 32, 113 - 120 (1998).

9. A. Chaturvedi and K. Surinder, Further remarks on estimating the reliability function of exponential distribution under type I and type II censorings, Brazilian Jour. Prob. Statist., 13, 29 - 39 (1999).

10. R. D. Gupta and R. C. Gupta, Estimation of $P\left(a^{\prime} x>b^{\prime} y\right)$ in the multivariate normal case, Statistics, 1, $91-97$ (1990).

11. Z. E. Jaheen, Bayesian approach to prediction with outliers from the Burr type X model, Microelectron. Rel., 35, 45 - 47 (1995).

12. Z. E. Jaheen, Empirical Bayes estimation of the reliability and failure rate functions of the Burr type X failure model, Journal of Applied Statistical Science, 3, 281 - 288 (1996).

13. N. L. Johnson, Letter to the editor, Technometrics, 17, p. 393 (1975).

14. N. L. Johnson and S. Kotz, Continuous Univariate Distributions - I. John Wiley and Sons, New York (1970).

15. N. L. Johnson, S. Kotz and N. Balakrishnan, Continuous Univariate Distribution, Vol. 1, 2nd Ed., New York, Wiley (1995).

16. G. D. Kelly, J. A. Kelly and W. R. Schucany, Efficient estimation of $P(Y<X)$ in the exponential case, Technometrics, 18, 359 - 360 (1976).

17. D. Kundu and M. Z. Raqab, Generalized Rayleigh distribution: different methods of estimations, Computational Statistics \& Data Analysis, 49, 187 - 200 (2005).

18. J. I. McCool, Inference on $P(X<Y)$ in the Weibull case, Commun. Statist. - Simul. Comp., 20 (1), $129-148$ (1991).

19. M. Z. Raqab, Order statistics from the Burr type X model, Computers Mathematics and Applications, 36, 111 - 120 (1998).

20. M. Z. Raqab and D. Kundu, Comparison of different estimators of $P[Y<X\}$ for a scaled Burr type X distribution, Commun. Statist. -Simul. Comp., 34, 465 - 483 (2005).

21. R. N. Rodriguez, A guide to Burr Type XII distributions, Biometrika, 64, 129 - 134 (1977).

22. V. K. Rohatgi, An Introduction to Probability Theory and Mathematical Statistics, John Wiley and Sons, New York, (1976).

23. H. A. Sartawi and M. S. Abu-Salih, Bayes prediction bounds for the Burr type X model, Communications in Statistics Theory and Methods, 20, 2307 - 2330 (1991).

24. S. K. Sinha, Reliability and Life Testing, Wiley Eastern Limited, New Delhi, (1986).

25. J. G. Surles and W. J. Padgett, Inference for $P(Y<X)$ in the Burr Type X model, Journal of Applied Statistical Science, 225 - 238 (1998).

26. J. G. Surles and W. J. Padgett, Inference for reliability and stress-strength for a scaled Burr Type X distribution, Lifetime Data Analysis, 7, 187 - 200 (2001).

27. J. G. Surles and W.J. Padgett, Some properties of a scaled Burr type X distribution, to appear in Journal of Statistical Planning and Inference, (2004).

28. R. K. Tyagi and S. K. Bhattacharya, A note on the MVU estimation of reliability for the Maxwell failure distribution, Estadistica, 41, 73 - 79(1989).

29. R. K. Tyagi, N. K. Singh, D. Kumar and S. K. Bhattacharya, Bayesian and non-Bayesian reliability analysis based on IEFD, ISIAM '92, Feb. 4-7, Deptt. of Mathematics, University of 
Roorkee, 227-232 (1993).

30. D. R. Wongo, Maximum likelihood methods for fitting the Burr Type XII distribution to multiply (progressively) censored life test data, Metrika, 40, 203 - 210(1993). 